\title{
Melatonin-induced methylation of the ABCG2/BCRP promoter as a novel mechanism to overcome multidrug resistance in brain tumour stem cells
}

\author{
V Martín *,1,2, A M Sanchez-Sanchez ${ }^{1,2}$, F Herrera ${ }^{3}$, C Gomez-Manzano ${ }^{4,5}$, J Fueyo ${ }^{4}$, M A Alvarez-Vega ${ }^{6}$, \\ I Antolín ${ }^{1}$ and C Rodriguez ${ }^{1,2}$ \\ ${ }^{1}$ Departamento de Morfología y Biología Celular, Facultad de Medicina, C/Julian Claveria 6, 33006 Oviedo, Spain; ${ }^{2}$ Instituto \\ Universitario de Oncología del Principado de Asturias (IUOPA), C/Julian Claveria 6, 33006 Oviedo, Spain; ${ }^{3}$ Cell and Molecular \\ Neuroscience Unit, Instituto de Medicina Molecular, Avenue Professor Egas Moniz, 1649-028 Lisboa, Portugal; ${ }^{4}$ Department of \\ Neuro-Oncology, The University of Texas M. D. Anderson Cancer Center, 1515 Holcombe Boulevard, Houston, TX 77030-4009, \\ USA; ${ }^{5}$ Department of Genetics, The University of Texas M. D. Anderson Cancer Center, 1515 Holcombe Boulevard, Houston, TX \\ 77030-4009, USA and ${ }^{6}$ Departamento de Neurocirugia, Hospital Central de Asturias (HUCA), C/Julian Claveria sn, 33006 Oviedo, \\ Spain
}

Background: Current evidence indicates that a stem cell-like sub-population within malignant glioblastomas, that overexpress members of the adenosine triphosphate-binding cassette $(A B C)$ family transporters, is responsible for multidrug resistance and tumour relapse. Eradication of the brain tumour stem cell (BTSC) compartment is therefore essential to achieve a stable and longlasting remission.

Methods: Melatonin actions were analysed by viability cell assays, flow cytometry, quantitative PCR for mRNA expression, western blot for protein expression and quantitative and qualitative promoter methylation methods.

Results: Combinations of melatonin and chemotherapeutic drugs (including temozolomide, current treatment for malignant gliomas) have a synergistic toxic effect on BTSCs and A172 malignant glioma cells. This effect is correlated with a downregulation of the expression and function of the ABC transporter ABCG2/BCRP. Melatonin increased the methylation levels of the ABCG2/ BCRP promoter and the effects on ABCG2/BCRP expression and function were prevented by preincubation with a DNA methyltransferase inhibitor.

Conclusion: Our results point out a possible relationship between the downregulation of ABCG2/BCRP function and the synergistic toxic effect of melatonin and chemotherapeutic drugs. Melatonin could be a promising candidate to overcome multidrug resistance in the treatment of glioblastomas, and thus improve the efficiency of current therapies.

Glioblastoma is the most common primary brain tumour. It is also one of the most lethal cancers, with an average life expectancy that remains less than 1 year. Treatment of glioblastoma patients is extremely challenging, as complete surgical resection of the tumour is very difficult and glioblastomas are refractory to current chemotherapy regimens. The development of novel therapeutic strategies for the treatment of malignant glioblastoma is therefore urgent. 
In recent years, numerous studies have reported the presence of stem cell-like cells in solid tumours including malignant glioblastomas (brain tumour stem cells, BTSC; Galli et al, 2004). These are defined as a built-in population of multidrug-resistant, pluripotent cells that can survive chemotherapy and repopulate the tumour after treatment. According to this hypothesis, therapeutic approaches that do not eradicate the BTSC compartment are likely to fail. They could kill the majority of tumour cells and induce a temporary regression, but then fail to prevent disease relapse. Consistently, there is an enrichment of BTSCs in response to chemotherapy treatments (Charles and Holland, 2009). In order to achieve a stable long-lasting remission of glioblastomas, there is necessary to find therapeutic approaches that target both the tumour bulk and the BTSC compartment.

Melatonin ( $N$-acetyl-5-methoxytryptamine) is an indolamine involved in the regulation of chronobiological rhythms and endocrine function (Lewy, 2007). However, melatonin also shows antiproliferative and cytotoxic effects on specific tumour types (Regelson and Pierpaoli, 1987; Maestroni, 1993; Sze et al, 1993) as well as direct and indirect antioxidant effects (Bonnefont-Rousselot and Collin, 2010). Long-term in vivo treatment with melatonin showed protection against several age-related events, including the incidence of malignant tumours, without noteworthy side effects (Tamarkin et al, 1981; Pierpaoli and Regelson, 1994). Moreover, concomitant administration of pharmacological doses of melatonin increases the therapeutic efficacy of cancer chemotherapy, particularly in patients with poor clinical status (Messina et al, 2010). This is consistent with clinical and experimental studies showing that other antioxidants enhance the cytotoxicity of chemotherapy (Ladas et al, 2004).

Our group has shown previously that melatonin inhibits rat glioblastoma cell proliferation both in vitro and in vivo by inhibiting key intracellular effectors such as PKC, Akt and NF-kB, effects that are also associated to the antioxidant properties of the indolamine (Martín et al, 2006). We also have previously showed melatonin to sensitise glioblastoma against TRAIL-induced cell death (Martín et al, 2010).

Here, we describe that melatonin increases the efficacy of antitumoural compounds in both BTSCs and glioblastoma cell lines. This effect is correlated with a decrease in the expression of the adenosine triphosphate-binding cassette (ABC) superfamily member ABCG2/BCRP, responsible for tumour resistance to some chemotherapeutic drugs.

\section{MATERIALS AND METHODS}

Cell culture and reagents. The human malignant glioblastoma cell lines A172, U87 and U373 (American Type Culture Collection, Manassas, VA, USA) were maintained in DMEM/F-12 (1:1, v/v) supplemented with $10 \%$ fetal bovine serum (FBS) and $1 \%$ antibiotic-antimycotic mixture containing $5.000 \mathrm{U} \mathrm{ml}^{-1}$ penicillin and $5.000 \mathrm{U} \mathrm{ml}^{-1}$ streptomycin. Neurospheroid cultures were established from acute cell dissociation of human glioblastoma post-surgical specimens, and maintained in DMEM/F12 medium supplemented with B27 (Invitrogen, Carlsbad, CA, USA), EGF and bFGF (20 $\mathrm{ng} \mathrm{ml}^{-1}$ each, Sigma-Aldrich, St Louis, MO, USA) according to the procedures described elsewhere (Galli et al, 2004; Singh et al, 2004). Neurospheroid cultures display a BTSC phenotype (self-renewal, proliferation, expression of stem cell markers, pluripotency and ability to form tumours in vivo; Jiang et al, 2007; Alonso et al, 2011).

Cell culture reagents were purchased from Sigma Chemical Co. (St Louis, MO, USA) except for FBS, which was obtained from Gibco (Invitrogen Life Technologies, Madrid, Spain). Culture flasks and dishes were acquired from Fisher Scientific (Madrid, Spain).
Melatonin and all other reagents were purchased from Sigma Chemical Co., unless otherwise indicated.

Cell viability assay. For dose-response curves, cells were platted on a 96-well dish at a density of $10^{4}$ cells per well and viability was determined by the MTT assay as previously described (Martín et al, 2010). Data were analysed using CalcuSyn software (Biosoft, Cambridge, UK), which calculates the $\mathrm{IC}_{50}$ for each drug and fits the curves to Chou-Talalay lines (Chou, 1998) in order to calculate the combination index (CI) at different 'effect' or 'fraction-affected' levels. A CI between 0.9 and 1.1 indicates an additive effect, a CI less than 0.9 indicates synergy, and a CI greater than 1.1 indicates antagonism.

Real-time quantitative PCR. Quantitative analysis of ABCG2, MDR1 and MRP1 mRNA levels was performed by the SYBR Green real-time PCR method using Green PCR Core Reagents (ABI systems, Foster City, CA, USA) in an AB7700 Real-Time System (ABI systems) as previously described (Martín et al, 2010). The primers used were the following: $5^{\prime}$-AGTTCCATGGCACTGGC CATA-3' (sense) and TCAGGTAGGCAATTGTGAGG (antisense) for ABCG2; 5'-CCCATCATTGCAATAGCAGG-3' (sense) and $5^{\prime}$-TGTTCAAACTTCTGCTCCTGA-3' (antisense) for MDR1; $5^{\prime}$-ATGGGGAAGGTGAAGGTCGG-3' (sense) and $5^{\prime}$-GACGGT GCCATGGAATTTGC-3' (antisense) for MRP1; and $5^{\prime}$-ATGGG GAAGGTGAAGGTCGG-3'(sense) and $5^{\prime}$-GACGGTGCCATG GAATTTGC-3'(antisense) for GAPDH. Each sample was tested in triplicate, and relative gene expression data were analysed by means of the $2^{-\triangle \mathrm{CT}}$ method.

Western blot. For protein expression, cells were lysed with icecold lysis buffer (150 mm NaCl, 1 mm EDTA, 1 mM EGTA, 1\% (v/v) Triton X-100, $2.5 \mathrm{~mm}$ sodium pyrophosphate, $1 \mathrm{~mm} \beta$-glycerophosphate, $1 \mathrm{~mm} \mathrm{Na}_{3} \mathrm{VO}_{4}, 1 \mu \mathrm{g} \mathrm{ml}{ }^{-1}$ leupeptin, $10 \mathrm{~nm} \mathrm{NaF}, 1 \mathrm{~mm}$ PMSF, $20 \mathrm{~mm}$ Tris-HCl pH 7.5). Thirty to fifty $\mu \mathrm{g}$ of total protein were separated by SDS-PAGE and transferred to polyvinylidene difluoride membranes (Amersham Bioscience, Pittsburgh, PA, USA). Blots were incubated with antibodies against ABCG2 (1:1000, Cell Signaling Technology, Beverly, MA, USA), and GAPDH-as a loading control $(1: 1000$, Santa Cruz Biotechnology, Santa Cruz, CA, USA). Blots were then incubated with horseradish peroxidase-conjugated secondary antibodies (anti-rabbit IgG peroxidase conjugated and anti-goat IgG peroxidase $1: 3000$, Calbiochem, La Jolla, CA, USA) and the reaction was visualised by means of enhanced-chemiluminescence detection reagents (Amersham Biosciences) following manufacturer's protocol.

Drug efflux studies. Mitoxantrone efflux studies were performed in order to evaluate the activity of $\mathrm{ABC}$ transporters. Briefly, cells were trypsinised and incubated for $30 \mathrm{~min}$ at $37^{\circ} \mathrm{C}$ and $5 \% \mathrm{CO}_{2}$ in complete medium containing $1 \mu \mathrm{M}$ mitoxantrone. Cells were then incubated for $1 \mathrm{~h}$ at $37^{\circ} \mathrm{C}$ in mitoxantrone-free medium to generate efflux histograms. Samples were analysed on a FACSCalibur flow cytometer (BD Biosciences, San Jose, CA, USA) equipped with a 635-nm (mitoxantrone excitation wavelength) red diode laser and a 561/16 band pass filter (FL4).

Qualitative analysis of ABCG2 promoter methylation (methylation-specific PCR (MSP) analysis). For MSP analysis, genomic DNA was obtained using the GeneElute Mammalian Genomic DNA miniprep Kit (Sigma-Aldrich, Milwaukee, WI, USA) according to manufacturer's protocol. Two-hundred nanogram of DNA per sample were modified using the Imprint DNA modification kit (Sigma-Aldrich) according to manufacturer's protocol. Primer pairs for MSP were designed on the basis of methylated and unmethylated DNA sequences in the promoter region as follows: $5^{\prime}$-GCGTTTCGGTTAGTGACGGC-3' (sense) and $5^{\prime}$-CCCGCCTCCGAAATCGAACG-3' (antisense) for methylation specific and $5^{\prime}$-TAGTTTTGTTGGTGGTTTAGTGT- ${ }^{\prime}$ 
(sense) and 5'-AACCCCAACTACCAAACCACA-3' (antisense) for unmethylation specific. Polymerase chain reaction was performed in $25 \mu \mathrm{l}$ reaction mixtures containing $50 \mathrm{ng}$ bisulphitemodified or -unmodified DNA, $20 \mathrm{~mm}$ Tris- $\mathrm{HCl} \mathrm{pH8.4,50} \mathrm{mм}$ $\mathrm{KCl}, 1.5 \mathrm{~mm} \mathrm{MgCl}_{2}, 240 \mu \mathrm{M}$ dNTPs, $0.4 \mu \mathrm{M}$ primers and $1.5 \mathrm{U}$ platinum Taq DNA polymerase (Invitrogen Life Technologies). Amplification was performed with an initial denaturation at $94{ }^{\circ} \mathrm{C}$ for $2 \mathrm{~min}$; 35 cycles of denaturation at $94^{\circ} \mathrm{C}$ for $30 \mathrm{~s}$, annealing at $60{ }^{\circ} \mathrm{C}$ for $60 \mathrm{~s}$ and extension at $72{ }^{\circ} \mathrm{C}$ for $30 \mathrm{~s}$; and a final extension step at $72{ }^{\circ} \mathrm{C}$ for $5 \mathrm{~min}$. Finally, PCR products were electrophoresed in a $2 \%$ agarose gel.

Quantitative analysis of ABCG2 promoter methylation. Quantitative analysis of ABCG2 promoter methylation was carried out using a Methyl-Profiler DNA Methylation qPCR System (SABiosciences, Quiagen, Valencia, CA, USA) according to manufacturer's protocol. Genomic DNA was extracted from BTSCs as described in the MSP section. Patient and normal brain samples from necropsies were obtained from the Leon Hospital Tumor Bank (Leon, Spain) after obtaining written informed consent according to protocols previously approved by the Clinic Research Ethics Committee of the institution and genomic DNA was extracted using the DNeasy Kit (Qiagen) according to the manufacturer's protocol. Briefly, $30 \mathrm{ng}$ of DNA from each sample were digested using either a methylation-sensitive or a methylation-dependent restriction enzyme. Following digestion, the remaining DNA was quantified by real-time PCR using primers that flank the promoter region of the $A B C G 2$ gene. The proportion of hypermethylated, intermediate methylated and unmethylated DNA in the samples was subsequently determined by comparing the amount of PCR product in each digest with a mock digest. A double digest was included in the experimental design in order to determine the background of the technique.

Data analysis. Experiments were repeated at least three times, and data were calculated as the average \pm s.e. Statistical comparisons were carried out using a one-way ANOVA followed by a StudentNewman-Keuls multiple range test. Statistical significance was accepted when $P<0.05$.

\section{RESULTS}

Melatonin enhances the toxicity of chemotherapeutic drugs on BTSCs. Previous reports suggest that antioxidants could enhance the efficacy of chemotherapy (Ladas et al, 2004). In order to test the effect of melatonin on the toxicity of chemotherapeutic drugs, three BTSC lines (NSC23, NSC7-2 and NSC11) were treated with different concentrations of the indole in combination with temozolomide, doxorubicin or mitoxantrone for $48 \mathrm{~h}$. Toxicity was then tested by the MTT assay, and the median-effect doses ( $\mathrm{IC}_{50}$ values) and $\mathrm{CI}$ values were calculated from dose-response curves as described in the Materials and Methods section.

We observed that melatonin greatly enhanced the toxicity of chemotherapeutic drugs. This was reflected in a significant decrease in $\mathrm{IC}_{50}$ values for each drug when it was combined with the indolamine (Figure 1A). Moreover, analysis of the CI values showed that co-incubation of melatonin and temozolomide, doxorubicin or mitoxantrone resulted in a synergistic toxic effect in the three BTSC lines tested (Figure 1B).

Melatonin decreases the expression of ABCG2/BCRP in BTSCs. Aberrant expression of $\mathrm{ABC}$ transporters is one of the main mechanisms responsible for multidrug resistance in cancer cells (Gottesman et al, 2002). Consistently, BTSCs showed much higher mRNA levels for ABCG2/BCRP and ABCB1/MDR1 than glioblastoma cell lines, whereas MRP1 mRNA levels were similar in all cells tested (Figure 2A). This was correlated with a higher resistance of BTSCs to temozolomide, doxorubicin and mitoxantrone, expressed as higher $\mathrm{IC}_{50}$ values (Figure $2 \mathrm{~B}$ ). Moreover, preincubation with verapamil, a well known $\mathrm{ABC}$ transporter inhibitor, decreased temozolomide, doxorubicin and mitoxantrone $\mathrm{IC}_{50}$ values in BTSCs (Figure 2C), supporting the relevance of ABCG2/BCRP and $\mathrm{ABCB} 1 / \mathrm{MDR} 1$ transporters in multidrug resistance of BTSCs.

A possible effect of melatonin on $\mathrm{ABC}$ transporter expression and function that could explain the synergy between melatonin and chemotherapeutic drugs was then evaluated. We found that melatonin induced a significant decrease in the mRNA levels of ABCG2/BCRP, but not ABCB1/MDR1 or MRP1, in BTSCs
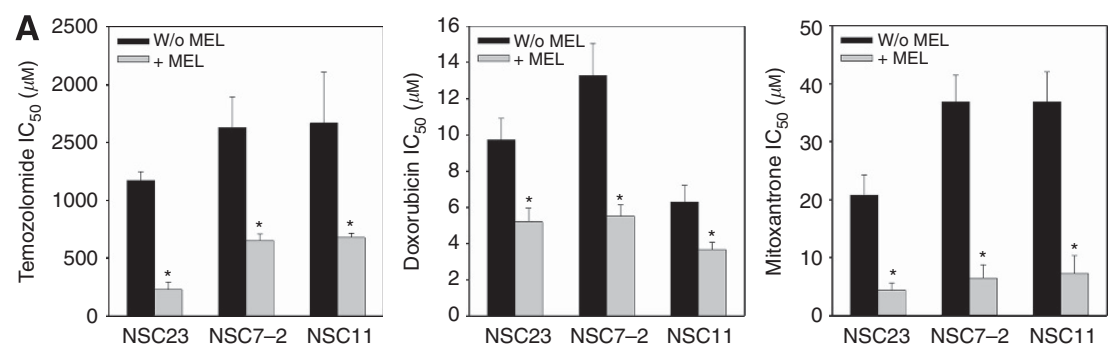

B

$\mathrm{Cl}$ values for chemotherapy plus melatonin

\begin{tabular}{|c|c|c|c|c|}
\hline \multirow[b]{2}{*}{ Drugs } & \\
\hline & $\mu \mathrm{M}$ & NSC23 & NSC7-2 & NSC11 \\
\hline \multirow{3}{*}{$\begin{array}{l}\text { Tenozolomide plus } \\
\text { melatonin } \\
\text { (ratio } 1: 1 \text { ) }\end{array}$} & $100: 100$ & 1.089 & 0.542 & 0.353 \\
\hline & $500: 500$ & 0.810 & 0.599 & 0.391 \\
\hline & $1000: 1000$ & 0.217 & 0.659 & 0.161 \\
\hline \multirow{3}{*}{$\begin{array}{c}\text { Doxorubicin plus } \\
\text { melatonin } \\
\text { (ratio } 1: 100)\end{array}$} & $1: 100$ & 0.113 & 0.897 & 0.735 \\
\hline & $5: 500$ & 0.170 & 0.357 & 0.342 \\
\hline & $10: 1000$ & 0.152 & 0.308 & 0.160 \\
\hline \multirow{3}{*}{$\begin{array}{l}\text { Mitoxantrone plus } \\
\text { melatonin } \\
\text { (ratio } 1: 100)\end{array}$} & $1: 100$ & 1.070 & 1.008 & 0.405 \\
\hline & $5: 500$ & 0.384 & 0.734 & 0.340 \\
\hline & $10: 1000$ & 0.156 & 0.206 & 0.160 \\
\hline
\end{tabular}

Figure 1. Synergistic toxic effect of melatonin and chemotherapy on BTSCs. (A), Cells were treated with temozolomide (0-2 mm), doxorubicin $(0-50 \mu \mathrm{M})$ and mitoxantrone $(0-50 \mu \mathrm{M})$ alone or in combination with melatonin (0-1 mM) for $48 \mathrm{~h}$ and viability was determined by MTT reduction. Results were analysed using CalcuSyn software to calculate $\mathrm{IC}_{50}$ values for each drug. ${ }^{\star} P<0.01$ vs chemotherapeutic drug alone. (B) Combination index $(\mathrm{Cl})$ values for the different drug combinations at a constant ratio calculated using CalcuSyn software. A $\mathrm{Cl}$ between 0.9 and 1.1 indicates an additive effect, a $\mathrm{Cl}$ less than 0.9 indicates synergy, and a $\mathrm{Cl}$ greater than 1.1 indicates antagonism. 

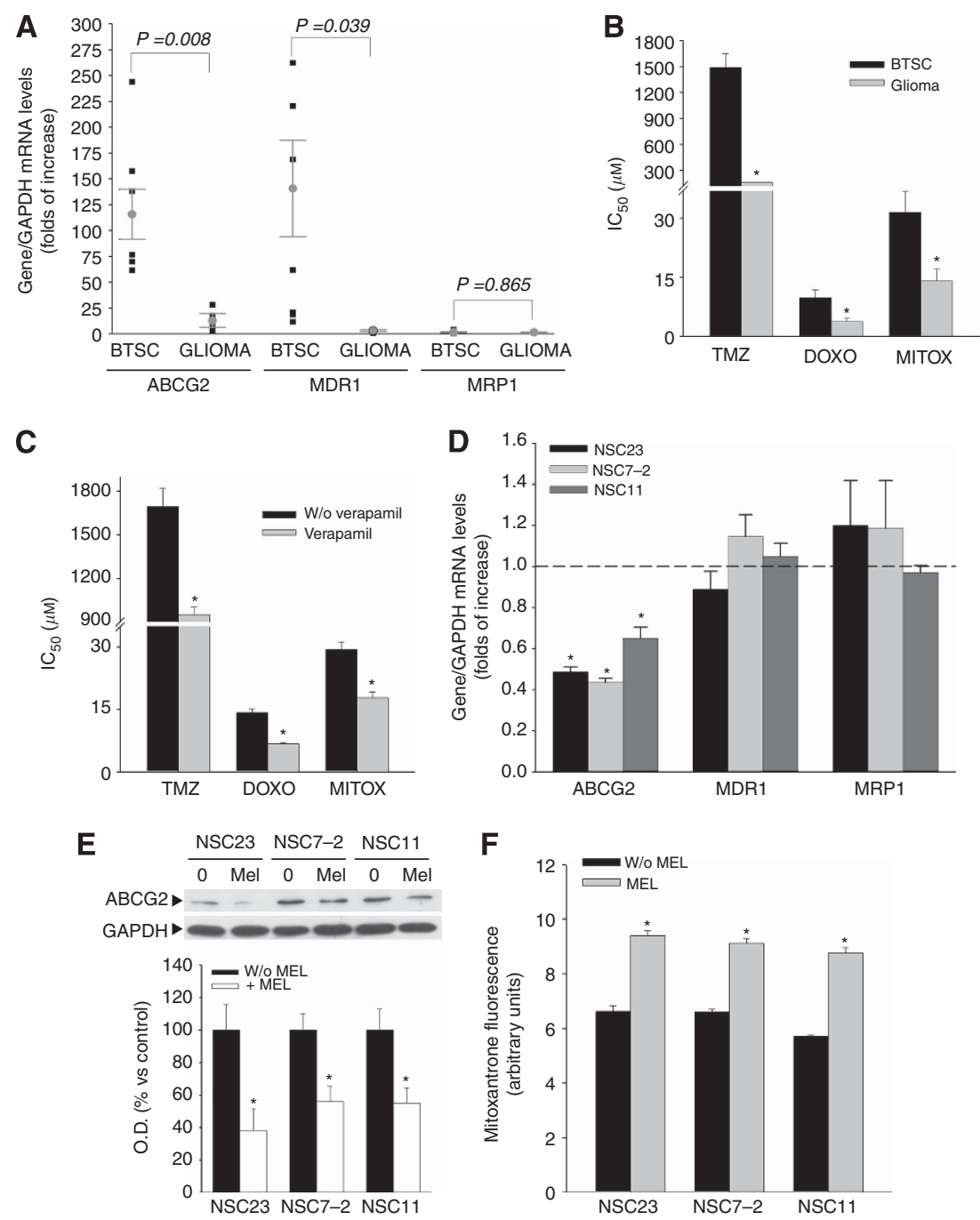

Figure 2. Melatonin regulation of $A B C G 2 / B C R P$ expression and function. (A) Relative mRNA expression levels of ABCG2/BCRP, ABCB1/MDR1 and MRP1 in both six BTSCs and three established glioblastoma cell lines (A172, U87, U373) as determined by quantitative PCR. (B) Comparison of $I_{50}$ values for temozolomide, doxorubicin and mitoxantrone in BTSCs and glioblastoma cell lines. Cells were treated with temozolomide $(0-2 \mathrm{~mm})$, doxorubicin $(0-50 \mu \mathrm{M})$ and mitoxantrone $(0-50 \mu \mathrm{M})$ for $48 \mathrm{~h}$ and viability was determined by MTT reduction. Results were analysed using CalcuSyn software to calculate $I_{50}$ values for each drug; ${ }^{*} P<0.01$ vs BTSCs. (C) IC $\mathrm{C}_{50}$ values for temozolomide, doxorubicin and mitoxantrone in BTSCs with or without the ABC transporters inhibitor verapamil. Cells were pre-treated with $10 \mu \mathrm{g} \mathrm{ml}^{-1}$ verapamil for $4 \mathrm{~h}$ before addition of chemotherapeutics as described earlier. Viability was determined by MTT reduction after $48 \mathrm{~h}$. Results were analysed using CalcuSyn software to calculate $\mathrm{IC}_{50}$ values for each drug; ${ }^{\star} P<0.01$ vs cells without verapamil. (D) mRNA expression levels of $A B C G 2 / B C R P, A B C B 1 / M D R 1$ and MRP1 after melatonin treatment in BTSCs as determined by quantitative PCR. Cells were treated with $1 \mathrm{~mm}$ melatonin for $24 \mathrm{~h}$. ${ }^{\star} P<0.01$ vs control group (vehicle-treated cells, dashed lined). (E) ABCG2/BCRP protein expression after melatonin treatment (1 mM for $24 \mathrm{~h}$ ) in BTSCs. Optical density reading values vs the loading control protein GAPDH are represented as percent of the control values. ${ }^{\star} P<0.01$ vs control group (vehicle-treated cells). (F) ABCG2 transporter activity represented as mitoxantrone (MTX) efflux after melatonin treatment (1 mM for $24 \mathrm{~h}$ ) in BTSCs. Fluorescence values with or without melatonin treatment are represented as arbitrary units. ${ }^{*} P<0.01$ vs control group (vehicle-treated cells).

(Figure 2D). This decrease was confirmed at the protein level (Figure 2E). Moreover, transporter activity was measured by a drug efflux assay using the autofluorescent properties of mitoxantrone. As showed in Figure 2F, cells treated with melatonin presented an increase in the intracellular content of the autofluorescent drug, indicating that the drug was not being exported by the $\mathrm{ABC}$ transporter.

Our results suggest that melatonin could overcome multidrug resistance in BTSCs through the downregulation of ABCG2/BCRP expression, leading to an increase in intracellular drug accumulation and the subsequent enhancement of cell toxicity.

Melatonin regulates methylation status of ABCG2/BCRP promoter. Several mechanisms have been reported to regulate the expression of ABCG2/BCRP in cancer stem cells, including epigenetic phenomena (To et al, 2006; Bleau et al, 2009). Consistently, we found that glioblastoma samples extracted from patients had lower methylation levels in the ABCG2 promoter than the normal brain (Figure 3A), a fact that could account for the higher ABCG2 expression found in these tumours. Moreover, treatment of BTSCs with melatonin induced a significant decrease in the levels of unmethylated promoter and a concomitant increase in the levels of hypermethylation and partial methylation (Figure 3B). This is consistent with the decrease in the expression of the transporter shown above (Figure 2D and E). The methylation of the ABCG2/BCRP promoter in BTSCs after melatonin treatment was also confirmed by MSP after bisulphite DNA modification, using methylation-specific primers. As showed 

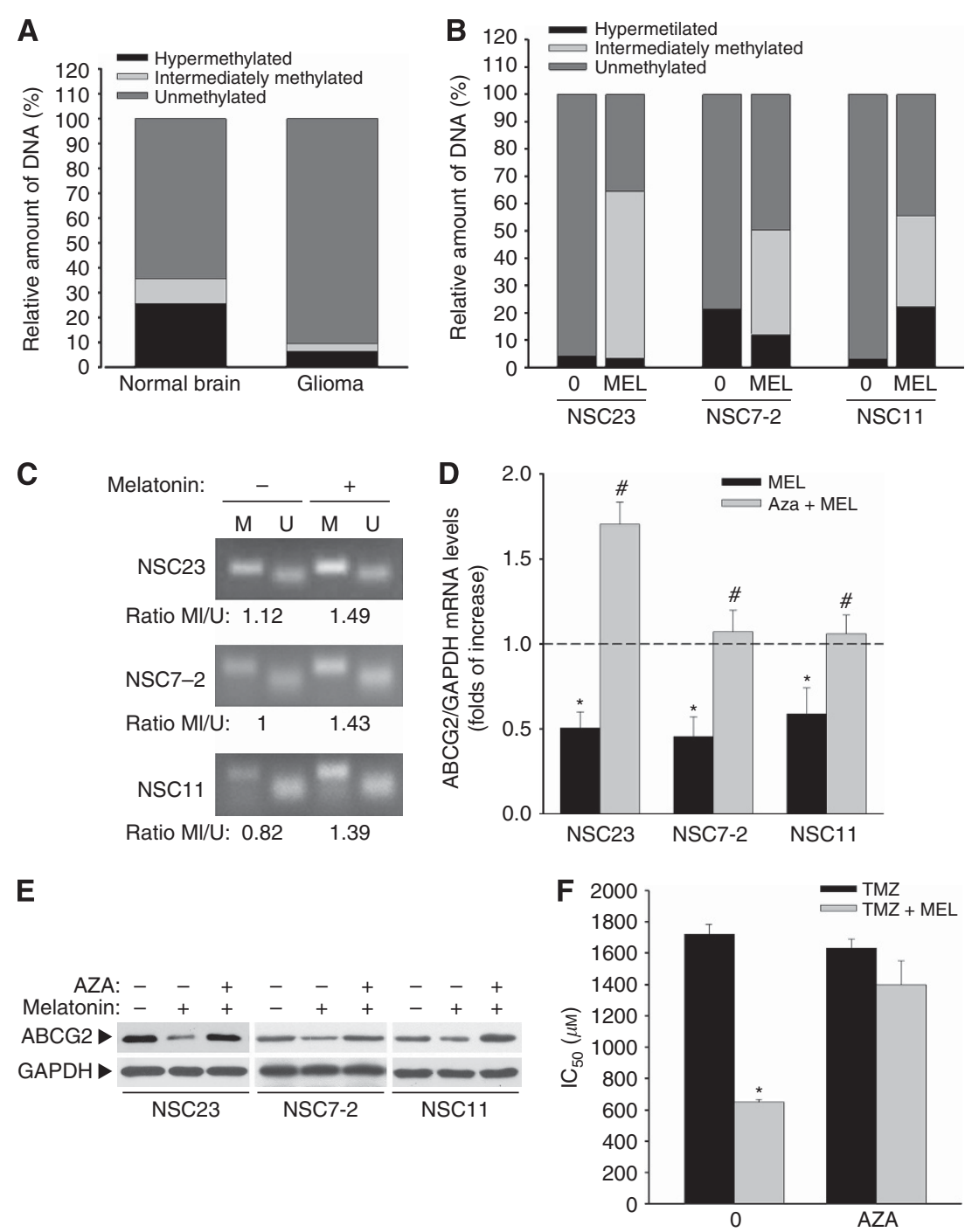

Figure 3. Melatonin regulates ABCG2/BCRP expression by inducing methylation of its promoter. (A) Quantitative analysis of $A B C G 2 / B C R P$ promoter methylation in normal brain and glioblastoma samples from patients. (B) Quantitative analysis of ABCG2/BCRP promoter methylation in BTSCs after melatonin treatment (1 mM for $24 \mathrm{~h}$ ). (C) MSP analysis of ABCG2/BCRP promoter region in BTSCs after melatonin treatment. PCR products recognising methylated $(M)$ and unmethylated $(U) C p G$ sites were analysed on $2 \%$ agarose gels. (D) mRNA expression levels of ABCG2/ BCRP in BTSCs after treatment with melatonin (1 mM for $24 \mathrm{~h}$ ) alone or in combination to the DNA methyltransferase inhibitor 5 -aza-dC (AZA, $1 \mu \mathrm{M})$. ${ }^{\star} P<0.01$ vs control group (vehicle-treated cells, dashed line). ${ }^{\#} P<0.01$ vs melatonin-treated cells. (E) ABCG2/BCRP protein expression in BTSCs after treatment with melatonin (1 mM for $24 \mathrm{~h}$ ) alone or in combination to the DNA methyltransferase inhibitor 5-aza-dC (AZA, $1 \mu \mathrm{M})$. (F) IC $\mathrm{C}_{50}$ values for temozolomide in the three BTSCs (NSC23, NSC7-2 and NSC11) alone or in combination to the DNA methyltransferase inhibitor 5-aza-dC (AZA, $1 \mu \mathrm{M})$. Viability was determined by MTT reduction after $48 \mathrm{~h}$ treatment. Results were analysed using CalcuSyn software to calculate $\mathrm{IC}_{50}$ values for each BTCSs and data are represented as the average. ${ }^{\star} P<0.01$ vs cells treated with temozolomide alone.

in Figure 3C, melatonin increased the levels of methylated promoter. DNA methyl-transferase inhibitor 5-azacitidine (AZA) prevents melatonin effect on the expression of ABCG2 at both the mRNA and protein levels (Figure 3D and E). Moreover, AZA preincubation also prevents melatonin synergistic effect on temozolomide toxicity in BTSCs expressed as $\mathrm{IC}_{50}$ (Figure $3 \mathrm{~F}$ ). These results strongly support that melatonin decreases transporter expression and BTSC resistance to chemotherapeutic drugs by inducing the methylation of the ABCG2/BCRP promoter.

Melatonin also decreases chemoresistance in glioblastoma cell lines. According to the cancer stem cells theory, the eradication of both tumour bulk and BTSCs is needed in order for glioblastoma treatments to succeed. In this regard, we found that melatonin not only potentiates chemotherapeutic drug cell death in BTSCs but also in a human malignant glioblastoma cell line (A172). Coincubation of A172 glioblastoma cells with melatonin significantly decreases the $\mathrm{IC}_{50}$ values of temozolomide, doxorubicin and mitoxantrone (Figure 4A), which results in a synergistic toxic effect as indicated by CI values (Figure 4B). Again, this effect is associated with a decrease in the expression and function of the ABCG2/BCRP transporter by melatonin (Figure 4C-E). An increase in promoter methylation by melatonin was also observed (Figure $4 \mathrm{~F}$ ), and this effect was also blocked by preincubation with AZA (Figure 4G), further supporting the epigenetic regulation of ABCG2/BCRP expression and function by melatonin.

In summary, our results strongly indicate that melatonin (1) enhances the efficacy of chemotherapeutic drugs, targeting both 
A

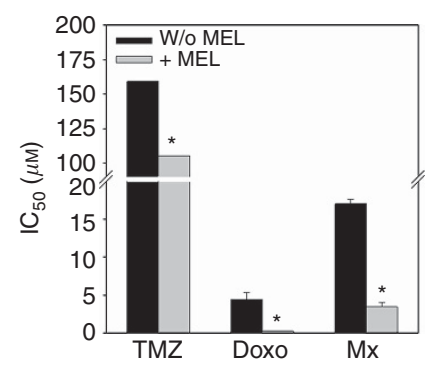

B

\begin{tabular}{ccc} 
& $\begin{array}{c}\text { values for chemotherapy plus } \\
\text { melatonin }\end{array}$ \\
\cline { 2 - 3 } Drugs & $\mu \mathrm{M}$ & $\mathrm{A} 172$ \\
\hline Temozolomide plus & $50: 50$ & 1.009 \\
melatonin & $100: 100$ & 0.593 \\
(ratio 1:1) & $500: 500$ & 0.164 \\
\hline Doxorubicin plus & $1: 100$ & 0.690 \\
melatonin & $5: 500$ & 0.440 \\
(ratio 1:100) & $10: 1000$ & 0.330 \\
\hline Mitoxantrone plus & $1: 100$ & 0.523 \\
melatonin & $5: 500$ & 0.293 \\
(ratio 1:100) & $10: 1000$ & 0.144 \\
\hline
\end{tabular}
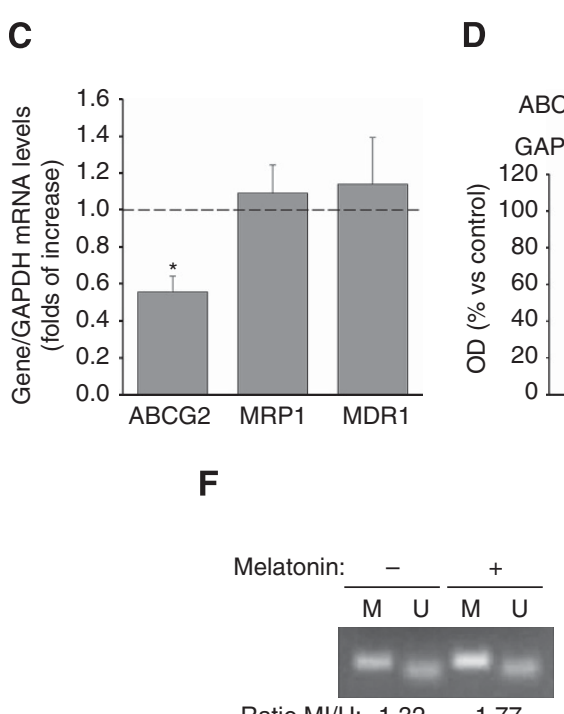

D

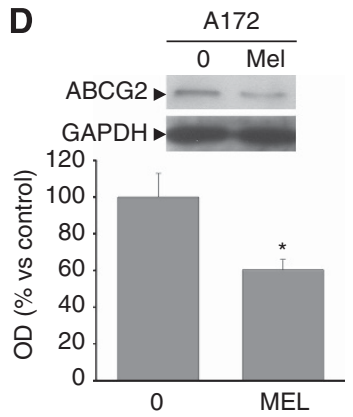

E

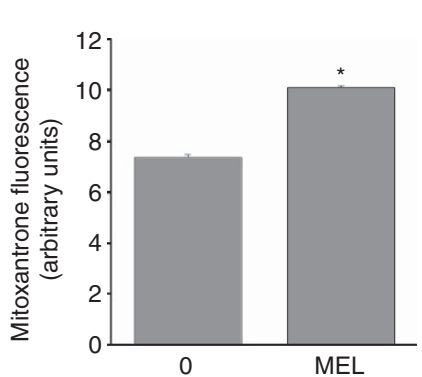

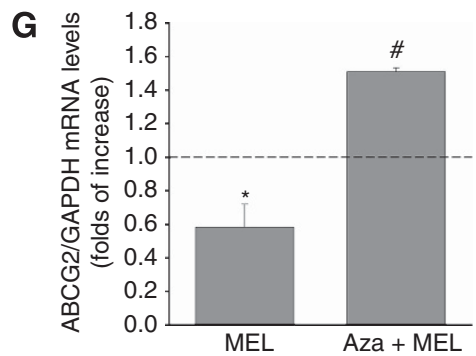

Figure 4. Melatonin also overcomes multidrug resistance in the A172 glioblastoma cell line. (A) Cells were treated with temozolomide (0-1 mM), doxorubicin $(0-10 \mu \mathrm{M})$ and mitoxantrone $(0-20 \mu \mathrm{M})$ alone or in combination with melatonin (0-1 mM) for $48 \mathrm{~h}$ and viability was determined by MTT reduction. Results were analysed using CalcuSyn software to calculate $I C_{50}$ values for each drug. ${ }^{*} P<0.01$ vs chemotherapeutic drug alone. (B) Combination index $(\mathrm{Cl})$ values for the different drug combinations at a constant ratio calculated using CalcuSyn software. A Cl between 0.9 and 1.1 indicates an additive effect, a $\mathrm{Cl}$ less than 0.9 indicates synergy and a $\mathrm{Cl}$ greater than 1.1 indicates antagonism. (C) mRNA expression levels of ABCG2/BCRP, ABCB1/MDR1 and MRP1 after melatonin treatment as determined by quantitative PCR. Cells were treated with $1 \mathrm{~mm}$ melatonin for $24 \mathrm{~h}$. ${ }^{\star} P<0.01$ vs control group (vehicle-treated cells, dashed line). (D) ABCG2/BCRP protein expression after melatonin treatment (1 mM for $24 \mathrm{~h}$ ). Optical density reading values vs the loading control protein GAPDH are represented as percent of the control values. ${ }^{\star} P<0.01$ vs control group (vehicle-treated cells). (E) ABCG2 transporter activity represented as mitoxantrone (MTX) efflux after melatonin treatment (1 mM for $24 \mathrm{~h}$ ).

Fluorescence values with or without melatonin treatment are represented as arbitrary units. ${ }^{\star} P<0.01$ vs control group (vehicle-treated cells). (F) MSP analysis of ABCG2/BCRP promoter region after melatonin treatment. PCR products recognising methylated (M) and unmethylated (U) CpG sites were analysed on $2 \%$ agarose gels. (G) mRNA expression levels of ABCG2/BCRP after treatment with melatonin ( $1 \mathrm{~mm}$ ) alone or in combination to the DNA methyltransferase inhibitor 5 -aza-dC (AZA, $1 \mu \mathrm{M})$. ${ }^{\star} P<0.01$ vs control group (vehicle-treated cells, dashed line). ${ }^{\#} P<0.01$ vs melatonin-treated cells.

BTSCs and the tumour bulk; and (2) regulates specifically the expression and function of the ABCG2/BCRP transporter by inducing the methylation of its promoter.

\section{DISCUSSION}

Discovery of a stem cell-like sub-population inside glioblastoma has highlighted the need for new therapeutic approaches that target both the tumour bulk and the BTSC compartment in order to achieve a stable and long-lasting remission. Data presented here showed that combination of melatonin with chemotherapeutics has a synergistic toxic effect both in BTSC and glioblastoma cell lines. Thus, melatonin increases toxicity of several drugs including temozolomide, current treatment for malignant glioblastoma.
Beneficial effects have been reported for melatonin when combined with a wide variety of drugs, including the increase in efficacy of chemotherapeutic drugs (Reiter et al, 2002). Synergistic toxic effects have been described in the combination of melatonin with several chemotherapeutics such as doxorubicin, tamoxifen, cisplatin or TNF and in several cancer types (Sánchez-Hidalgo et al, 2012). In this regard, we have previously showed melatonin to sensitise glioblastoma against TRAIL-induced cell death (Martín et al, 2010).

The variety of drugs that have been described to be potentiated by melatonin suggests an effect on cellular multidrug resistance mechanisms. Multidrug resistance is the principal mechanism by which many cancers develop resistance to chemotherapeutics and is a major factor in the failure of many forms of chemotherapy. Aberrant expression of $\mathrm{ABC}$ transporters is one of the main 
mechanisms responsible for multidrug resistance in cancer cells (Gottesman et al, 2002). Overexpression of several ABC transporters, including $\mathrm{ABCB} 1 / \mathrm{MDR} 1, \mathrm{MRP}$ and ABCG2/BCRP, results in an ATP-driven efflux of drugs from cancer cells, thereby leading to decreased intracellular drug concentrations and lower toxicity. Stem cells, both normal and tumoural, show higher expression levels of several ABC transporters (Jin et al, 2008). Consistently, our results showed overexpression of $A B C G 2 / B C R P$ and $A B C B 1 /$ MDR1 in BTSCs compared with glioblastoma cell lines that was correlated with a higher resistance of BTSCs to temozolomide, doxorubicin and mitoxantrone. Moreover, our data showed that verapamil decreases temozolomide, doxorubicin and mitoxantrone resistance in BTSCs, supporting the relevance of $\mathrm{ABC}$ transporters in multidrug resistance of BTSCs. Thus, the observed decrease in expression and function of ABCG2/BCRP after melatonin treatment in both BTSCs and glioblastoma cell lines could be responsible for the potentiation of chemotherapeutic drug cytotoxicity. Both mitoxantrone and doxorubicin have been widely described as substrates for the ABCG2/BCRP transporter, which actively exports drugs from the intracellular to the extracellular space. Although there are no direct evidences of temozolomide as a substrate for the ABCG2/BCRP transporter, some indirect evidence suggests that temozolomide is also a substrate of this transporter (Persson and Weiss, 2009). As far as we know, this is the first report showing any effect of melatonin on $\mathrm{ABC}$ transporter expression and function.

Several mechanisms have been reported to regulate the expression of ABCG2/BCRP in cancer stem cells, including epigenetic phenomena (To et al, 2006; Bleau et al, 2009). An active $\mathrm{CpG}$ island has been described within the proximal ABCG2/ BCRP promoter region, which inhibits the expression of the transporter when it is methylated (To et al, 2006). Consistently, we found that glioblastoma tumour had lower methylation levels in the ABCG2 promoter than the normal brain, a fact that could account for the higher ABCG2 expression found in these tumours. Treatment of BTSCs and glioblastoma cell lines with melatonin induced a significant increase in the promoter methylation levels that seemed to be responsible for the decrease in the expression of the transporter and so responsible for the synergistic toxic effect of melatonin in combination with chemotherapeutic drugs. This is one of the first reports showing any epigenetic control of gene expression induced by melatonin. Only a few recent reports suggest a possible link between the indolamine and the epigenetic phenomena by modulating the expression and recruitment of histone deacetylases (HDACs) and by inhibiting sirtuins, HDACs that have been described to be overexpressed in tumour cells (Korkmaz et al, 2012). However, althoguh these reports are based on indirect evidence, we demonstrate a clear effect of melatonin on the methylation of the $\mathrm{ABC}$ transporter. Our results showing that AZA prevents melatonin effects on ABCG2/BCRP expression suggest an effect of the indolamine on DNA methyltransferase activity rather than on HDACs and chromatin remodeling.

In summary, our results strongly indicate that melatonin enhances the efficacy of chemotherapeutic drugs, targeting both BTSCs and the tumour bulk due to the regulation of the expression and function of the ABCG2/BCRP transporter by inducing the methylation of its promoter. We also have previously described that melatonin inhibits the proliferation of glioblastoma cells both in vitro and in vivo (Martín et al, 2006) and we and others have also shown that melatonin is cytotoxic for some particular cancer cells (Sánchez-Sánchez et al, 2011; García-Santos et al, 2012). Therefore, melatonin could target directly tumour cells by inhibiting their proliferation or killing them, and simultaneously enhance the effect of chemotherapy. Our results point out melatonin as a promising candidate for the treatment of malignant glioblastoma. Further studies should be carried out in order to elucidate the role of melatonin in epigenetic control of gene expression and in the mechanisms by which it enhances the toxicity of chemotherapeutic drugs.

\section{ACKNOWLEDGEMENTS}

We thank Marina Gómez-Lobo for her excellent technical assistance. This work was supported by Ministry of Science and Innovation (SAF2010-21729) and FICYT Grants (FC11-COF1132) to Carmen Rodriguez. Ana Sanchez-Sanchez was supported by a FICYT fellowship (BP06-161). Vanesa Martin acknowledges the support by the Juan de la Cierva Program from the Ministry of Science and Education.

\section{REFERENCES}

Alonso MM, Diez-Valle R, Manterola L, Rubio A, Liu D, Cortes-Santiago N, Urquiza L, Jauregi P, Lopez de Munain A, Sampron N, Aramburu A, Tejada-Solís S, Vicente C, Odero MD, Bandrés E, García-Foncillas J, Idoate MA, Lang FF, Fueyo J, Gomez-Manzano C (2011) Genetic and epigenetic modifications of Sox 2 contribute to the invasive phenotype of malignant gliomas. PLoS One 6(11): e26740.

Bleau AM, Huse JT, Holland EC. (2009) The ABCG2 resistance network of glioblastoma. Cell Cycle 8(18): 2936-2944.

Bonnefont-Rousselot D, Collin F. (2010) Melatonin: action as antioxidant and potential applications in human disease and aging. Toxicology 278(1): $55-67$.

Charles N, Holland EC. (2009) Brain tumor treatment increases the number of cancer stem-like cells. Expert Rev Neurother 9(10): 1447-1449.

Chou TC. (1998) Drug combinations: from laboratory to practice. J Lab Clin Med 132: 6-8.

Galli R, Binda E, Orfanelli U, Cipelletti B, Gritti A, De Vitis S, Fiocco R, Foroni C, Dimeco F, Vescovi A. (2004) Isolation and characterization of tumorigenic, stem-like neural precursors from human glioblastoma. Cancer Res 64: 7011-7021.

García-Santos G, Martin V, Rodríguez-Blanco J, Herrera F, Casado-Zapico S, Sánchez-Sánchez AM, Antolín I, Rodríguez C. (2012) Fas/Fas ligand regulation mediates cell death in human Ewing's sarcoma cells treated with melatonin. Br J Cancer 106(7): 1288-1296.

Gottesman MM, Fojo T, Bates SE. (2002) Multidrug resistance in cancer: role of ATP-dependent transporters. Nat Rev Cancer 2(1): 48-58.

Jiang H, Gomez-Manzano C, Aoki H, Alonso MM, Kondo S, McCormick F, Xu J, Kondo Y, Bekele BN, Colman H, Lang FF, Fueyo J. (2007) Examination of the therapeutic potential of Delta-24-RGD in brain tumor stem cells: role of autophagic cell death. J Natl Cancer Inst 99(18): 1410-1414.

Jin F, Zhao L, Zhao HY, Guo SG, Feng J, Jiang XB, Zhang SL, Wei YJ, Fu R, Zhao JS. (2008) Comparison between cells and cancer stem-like cells isolated from glioblastoma and astrocytoma on expression of antiapoptotic and multidrug resistance-associated protein genes. Neuroscience 154(2): 541-550.

Korkmaz A, Rosales-Corral S, Reiter RJ. (2012) Gene regulation by melatonin linked to epigenetic phenomena. Gene 503(1): 1-11.

Ladas EJ, Jacobson JS, Kennedy DD, Teel K, Fleischauer A, Kelly KM. (2004) Antioxidants and cancer therapy: a systematic review. J Clin Oncol 22(3): $517-528$.

Lewy AJ. (2007) Melatonin and human chronobiology. Cold Spring Harb Symp Quant Biol 72: 623-636.

Maestroni GJ. (1993) The immunoneuroendocrine role of melatonin. J Pineal Res 14(1): 1-10.

Martín V, Herrera F, Carrera-Gonzalez P, García-Santos G, Antolín I, Rodriguez-Blanco J, Rodriguez C. (2006) Intracellular signaling pathways involved in the cell growth inhibition of glioma cells by melatonin. Cancer Res 66(2): 1081-1088.

Martín V, García-Santos G, Rodriguez-Blanco J, Casado-Zapico S, SanchezSanchez A, Antolín I, Rodriguez C. (2010) Melatonin sensitizes human malignant glioma cells against TRAIL-induced cell death. Cancer Lett 287(2): 216-223.

Messina G, Lissoni P, Marchiori P, Bartolacelli E, Brivio F, Magotti L. (2010) Enhancement of the efficacy of cancer chemotherapy by the pineal hormone melatonin and its relation with the psychospiritual status of cancer patients. J Res Med Sci 15(4): 225-228. 
Persson AI, Weiss WA. (2009) The side story of stem-like glioma cells. Cell Stem Cell 4(3): 191-192.

Pierpaoli W, Regelson W. (1994) Pineal control of aging: effect of melatonin and pineal grafting on aging mice. Proc Natl Acad Sci USA 91: 787-791.

Regelson W, Pierpaoli W. (1987) Melatonin: a rediscovered antitumor hormone? Its relation to surface receptors; sex steroid metabolism, immunologic response, and chronobiologic factors in tumor growth and therapy. Cancer Invest 5(4): 379-385.

Reiter RJ, Tan DX, Sainz RM, Mayo JC, Lopez-Burillo S. (2002) Melatonin: reducing the toxicity and increasing the efficacy of drugs. J Pharm Pharmacol 54(10): 1299-1321.

Sánchez-Hidalgo M, Guerrero JM, Villegas I, Packham G, de la Lastra CA (2012) Melatonin, a natural programmed cell death inducer in cancer. Curr Med Chem 19(22): 3805-3821.

Sánchez-Sánchez AM, Martín V, García-Santos G, Rodríguez-Blanco J, Casado-Zapico S, Suarez-Garnacho S, Antolín I, Rodriguez C. (2011) Intracellular redox state as determinant for melatonin antiproliferative vs cytotoxic effects in cancer cells. Free Radic Res 45(11-12): 1333-1341.
Singh SK, Hawkins C, Clarke ID, Squire JA, Bayani J, Hide T, Henkelman RM, Cusimano MD, Dirks PB. (2004) Identification of human brain tumour initiating cells. Nature 432(7015): 396-401.

Sze SF, Ng TB, Liu WK. (1993) Antiproliferative effect of pineal indoles on cultured tumor cell lines. J Pineal Res 14(1): 27-33.

Tamarkin L, Cohen M, Rosell D, Reichert C, Lippman M, Chabner B. (1981) Melatonin inhibition and pinealectomy enhacement of dimethylbenz(a) anthracene-induced mammary tumors in the rat. Cancer Res 41: 4432-4436.

To KK, Zhan Z, Bates SE (2006) Aberrant promoter methylation of the ABCG2 gene in renal carcinoma. Mol Cell Biol 26(22): 8572-8585.

This work is published under the standard license to publish agreement. After 12 months the work will become freely available and the license terms will switch to a Creative Commons AttributionNonCommercial-Share Alike 3.0 Unported License. 